\title{
A NOTE ON TRANSLITERATION AND TRANSLATION
}

We intend this volume for specialists and nonspecialists alike, and thus the essays transliterate Russian names in their most familiar English spellings, for example, "Solovyov" and "Tolstoy." Russian quotations in the text, as well as all bibliographical information, use the modified Library of Congress system of transliteration except when they are given in Cyrillic. All material quoted from Russian sources is also given in English translation; unless otherwise noted, the translations are by the authors. 

Sexuality and the Body in Russian Culture 
